\title{
A QUESTION CONCERNING POSITIVE TYPE POLYNOMIALS
}

\author{
HENRY TEICHER
}

1. The problem. In [1], the following problem is posed: Let $0<A<1$. Find the smallest integer $n$ for which there exist $n$ real numbers $a_{1}, a_{2}, \cdots, a_{n}$ such that the polynomial $\left(y^{2}-2 A y+1\right)$ - $\prod_{i=1}^{n}\left(y+a_{i}\right)$ has all its coefficients non-negative. If $N$ is such an integer, does there exist some real number $a$ such that $\left(y^{2}-2 A y+1\right)$ $\cdot(y+a)^{N}$ has only non-negative coefficients. ${ }^{1}$

In the process of giving the solution, we recast the problem slightly and put it into relief against several similar problems.

Let $P_{(n)}^{+}$denote the class of positive type polynomials, [2], of degree $n$, that is, $P_{(n)}^{+}=\left\{P_{n}(x)\right\}=\left\{\sum_{j=0}^{n} a_{j} x^{j}, a_{j} \geqq 0, j=0,1, \cdots, n\right\}$. Here, we suppose, in addition, without loss of generality, that $a_{0}>0$, $a_{n}=1$. Define $\mathcal{P}^{+}=\mathcal{P}_{0}^{+}=\bigcup_{n=1}^{\infty} \mathcal{P}_{(n)}^{+}$. Further, let $\mathcal{P}_{1}^{+}$(respectively, $\mathcal{P}_{2}^{+} ; \mathcal{P}_{3}^{+}$) denote the class of polynomials, all of whose roots are real and negative (respectively, are equal and negative; have negative real part). What is the smallest integer $N_{j}=N_{j}(\epsilon)$ such that $\left(x^{2}-x+\epsilon\right) Q_{N_{j}}(x) \in \mathcal{P}^{+}$where $\epsilon>1 / 4$ and $Q_{N_{j}}(x)$ is a polynomial of degree $N_{j}$ which belongs to $\rho_{j}^{+}$? Here, $j$ takes any of the values $0,1,2,3$. If $j=0$, the answer is contained in the statement and proof of Theorem 1 of [2]. For $j=1$, the smallest integer $n$ (namely $N_{1}$ ) for which $\left(x^{2}-x+\epsilon\right) \prod_{i=1}^{n}\left(x+b_{i}\right) \in \mathcal{P}^{+}$is exactly the integer $N$ mentioned earlier, as the correspondence $y \rightarrow 2 A x, \epsilon \rightarrow\left(4 A^{2}\right)^{-1}, a_{i} \rightarrow A b_{i}$ shows. Clearly, $b_{i} \geqq 0$, all $i$ and when $n=N_{1}, b_{i}>0, i=1,2, \cdots, N_{1}$ For $j=2$, the above question is intimately related to the second part of the research problem while for $j=3$, the connection is more remote.

All of these questions are rendered easier by an inversion, that is, the fixing of $n$ and the quest for the smallest $\epsilon=\epsilon_{n}$ such that

$$
\left(x^{2}-x+\epsilon\right) Q_{n}(x) \in \rho^{+} .
$$

It is then a simple matter to express conditions for (1.1) in terms of the coefficients of $Q_{n}(x)$. The difficulties arise in fulfilling these and simultaneously insuring that $Q_{n}(x) \in \mathcal{P}_{j}^{+}$. Fortunately, when $j=1$ (which will henceforth be supposed), necessary conditions that

Presented to the Society, February 28, 1959; received by the editors May 16, 1958 and, in revised form, September 24, 1958.

1 A third part of the problem asks for a generalization in which the factor $\left(y^{2}-2 A y+1\right)$ is replaced by an arbitrary polynomial (without positive roots). This appears to be of a different order of difficulty. 
$Q_{n}(x)=\prod_{i=1}^{n}\left(x+b_{i}\right) \in \mathcal{P}_{1}^{+}$are sufficient to resolve the problem. Writing $b=\left(b_{1}, \cdots, b_{n}\right)$ any pair $(\epsilon, b)$ satisfying (1.1) will be called a "real solution." $C_{n, j}$ will denote the combinatorial symbol.

2. The solution. Define $Q_{n}(x)=\sum_{j=0}^{n} C_{n, j} p_{j} x^{n-j}, p_{0}=1$. For (1.1) to hold, it is evidently necessary and sufficient that

$$
\begin{array}{rr}
C_{n, j+1} p_{j+1}-C_{n, j} p_{j}+\epsilon C_{n, j-1} p_{j-1} \geqq 0, & j=1, \cdots, n-1, \\
n p_{1} \geqq 1, & \epsilon n p_{n-1} \geqq p_{n} .
\end{array}
$$

Define further $r_{j}=p_{j} / p_{j-1}, j=1,2, \cdots, n$. The preceding equations take the form

$$
\begin{gathered}
\frac{n-j}{j+1} r_{j+1}+\frac{\epsilon j}{n-j+1} \frac{1}{r_{j}} \geqq 1, \quad j=1,2, \cdots, n-1, \\
r_{1} \geqq n^{-1}, \quad r_{n} \leqq \epsilon n .
\end{gathered}
$$

A necessary condition, [3 ], that in addition, $Q_{n}(x)=\prod_{i=1}^{n}\left(x+b_{i}\right)$ for real $b_{i}$, that is, $p_{j}=\left(C_{n, j}\right)^{-1} \sum b_{i_{1}} b_{i_{2}} \cdots b_{i_{j}}$ where the summation is over indices $1 \leqq i_{1}<i_{2}<\cdots<i_{j} \leqq n$, is conveniently expressed as

$$
r_{1} \geqq r_{2} \geqq \cdots \geqq r_{n} \text {. }
$$

The equality of any pair of the $r$ 's, say, $r_{i}=r_{j}=r$ implies the equality of all intervening $r$ 's (if any) and hence $b_{1}=b_{2}=\cdots=b_{n}=r$. Conversely, $b_{1}=b_{2}=\cdots=b_{n}=b$ clearly implies $r_{1}=r_{2}=\cdots=r_{n}$ $=b$. These facts will be used later.

It follows directly from (2.2) and (2.3) that any real solution must satisfy also

$$
r_{j+1}^{2}-\frac{j+1}{n-j} r_{j+1}+\frac{\epsilon j(j+1)}{(n-j)(n-j+1)} \geqq 0, j=1, \cdots, n-1
$$

that is, for $j=1,2, \cdots, n-1$

$$
\begin{aligned}
r_{j+1} & \leqq \frac{j+1}{2(n-j)}\left[1-\left(1-\frac{4 \epsilon j(n-j)}{(j+1)(n-j+1)}\right)^{1 / 2}\right] \\
& =\bar{r}_{j+1}(\epsilon) \quad \text { (say) }
\end{aligned}
$$

or

$$
r_{j+1} \geqq \frac{j+1}{2(n-j)}\left[1+\left(1-\frac{4 \epsilon j(n-j)}{(j+1)(n-j+1)}\right)^{1 / 2}\right]=r_{j+1}^{+}(\epsilon) \quad \text { (say). }
$$

We pause now in the general line of reasoning to establish two crucial results. Let $\epsilon$ be such that $r_{j}^{-}=r_{j}^{-}(\epsilon)$ and $r_{j}^{+}=r_{j}^{+}(\epsilon)$ are real valued for $j=2,3, \cdots, n$ : 
A. (a) If $n$ is odd and $\epsilon \leqq(n+3)^{2} / 4(n+1)^{2}$ then $r_{j+1}^{-1}(\epsilon) \leqq r_{j}^{+}(\epsilon)$ for $j=1,2, \cdots, n-1$. Further, for this range of $\epsilon$, equality holds only if $j=(n+1) / 2$ and $\epsilon=(n+3)^{2} / 4(n+1)^{2}$.

(b) If $n$ is even and $\epsilon \leqq(n+4) / 4 n$ then $r_{\bar{j}+1}(\epsilon) \leqq r_{j}^{+}(\epsilon)$ for $j=1,2, \cdots, n-1$. Further, for this range of $\epsilon$, equality holds only if $j=n / 2$ or $(n+2) / 2$, and $\epsilon=(n+4) / 4 n$.

PROOF. $r_{j+1}^{-} \leqq r_{j}^{+}$is tantamount to

$$
\begin{aligned}
& \frac{(j+1)(n-j+1)-\left((j+1)^{2}(n-j+1)^{2}-4 \epsilon j(j+1)(n-j)(n-j+1)\right)^{1 / 2}}{2(n-j)(n-j+1)} \\
& \leqq \frac{j(n-j+2)+\left(j^{2}(n-j+2)^{2}-4 \epsilon j(j-1)(n-j+1)(n-j+2)\right)^{1 / 2}}{2(n-j+1)(n-j+2)},
\end{aligned}
$$

or

$$
\begin{aligned}
& (n+1)-\left((j+1)^{2}(n-j+1)^{2}-4 \epsilon j(j+1)(n-j)(n-j+1)\right)^{1 / 2} \\
& \leqq(n-j)\left(j^{2}-4 \epsilon j(j-1)(n-j+1)(n-j+2)^{-1}\right)^{1 / 2} .
\end{aligned}
$$

If $j$ is such that the left hand side (L.H.S.) is negative, the preceding is automatically true; otherwise it is equivalent to

$$
\begin{aligned}
4 \epsilon j(n-j+1)-j(n-j+2) & \\
& \leqq\left(j^{2}(n-j+2)^{2}-4 \epsilon j(j-1)(n-j+1)(n-j+2)\right)^{1 / 2} .
\end{aligned}
$$

Again, this is trivially true if the L.H.S. is negative and otherwise equivalent to $4 \epsilon j(n-j+1) \leqq(j+1)(n-j+2)$ or

$$
j(n-j+1) \leqq(n+2)(4 \epsilon-1)^{-1} .
$$

If now $n$ is odd, rewrite (2.6) in the form

$$
0 \leqq\left(j-\frac{n+1}{2}\right)^{2}+\frac{n+2}{4 \epsilon-1}-\frac{(n+1)^{2}}{4}
$$

and, retracing the steps, this yields (a).

On the other hand if $n$ is even, rewrite (2.6) as

$$
0 \leqq\left(j-\frac{n}{2}\right)\left(j-\frac{n+2}{2}\right)+(n+2)\left[\frac{1}{4 \epsilon-1}-\frac{n}{4}\right]
$$

from which (b) is deduced.

B. (a) If $r_{j+1} \leqq r_{\overline{j+1}}$, then $r_{j} \leqq r_{\bar{j}+1}, j=1,2 \cdots n-1$.

(b) If, for $n$ odd, $\epsilon \leqq(n+3)^{2} / 4(n+1)^{2}$ or for $n$ even, $\epsilon \leqq(n+4) / 4 n$ then $r_{j} \geqq r_{j}^{+}$implies $r_{j+1} \geqq r_{j}^{-}, j=1,2 \cdots n-1$ (equality for $n$ odd only if $j=(n+1) / 2$ and $\epsilon=(n+3)^{2} / 4(n+1)^{2}$; equality for $n$ even only if $j=n / 2$ or $(n+2) / 2$, and $\epsilon=(n+4) / 4 n)$. 
Proof: Noting from (2.4) that

$$
\overline{r_{j+1}} \cdot r_{j+1}^{+}=\frac{\epsilon j(j+1)}{(n-j)(n-j+1)}, \quad i=1,2 \cdots n-1,
$$

we see from (2.2) and the hypothesis of (a) that

$$
\begin{aligned}
r_{j} & \leqq \epsilon j(n-j+1)^{-1}\left[1-\frac{n-j}{j+1} \overline{r_{j+1}}\right]^{-1} \\
& =\epsilon j(n-j+1)^{-1}\left[\frac{1}{2}\left(1+\left(1-\frac{4 \epsilon j(n-j)}{(j+1)(n-j+1)}\right)^{1 / 2}\right)\right]^{-1} \\
& =\frac{j+1}{2(n-j)}\left[1-\left(1-\frac{4 \epsilon j(n-j)}{(j+1)(n-j+1)}\right)^{1 / 2}\right]=\overline{r_{j+1}}
\end{aligned}
$$

To prove (b), we note from $A$ that $\epsilon j /(n-j+1) \cdot 1 / r_{\bar{j}+1}>\epsilon j /(n-j+1)$ $\cdot 1 / r_{j}^{+}$, or equivalently

$$
\begin{aligned}
1-\frac{\epsilon j}{(n-j+1) r_{j}^{+}} & >1-\frac{1}{2}\left[1+\left(1-\frac{4 \epsilon j(n-j)}{(j+1)(n-j+1)}\right)^{1 / 2}\right] \\
& =\frac{n-j}{j+1} r_{j+1} .
\end{aligned}
$$

Hence, the hypothesis of (b) and (2.2) imply

$$
\frac{n-j}{j+1} r_{j+1} \geqq 1-\frac{\epsilon j}{(n-j+1) r_{j}^{+}}>\frac{n-j}{j+1} \overline{r_{j+1}}
$$

which yields the conclusion of (b).

In returning to the problem as a whole, we discard the possibility that $r_{j}^{-}(\epsilon)$ and $r_{j}^{+}(\epsilon)$ are complex valued since this occurs only for values of $\epsilon$ larger than those for which we establish solutions. Also, we must separate the case of odd $n$ from that of even $n$. Suppose first that $n=2 k+1$ where $k$ is a non-negative integer. We shall show that $\epsilon_{n}^{\prime}=(n+3)^{2} / 4(n+1)^{2}$ is the smallest value of $\epsilon$ for which there exist real numbers $r_{1}, r_{2} \cdots r_{n}$ satisfying (2.2), (2.2)' and (2.3). As a consequence, no real solution is possible for $\epsilon<\epsilon_{n}^{\prime}$. We shall also find a (unique) real solution for $\epsilon=\epsilon_{n}^{\prime}$. To this end, we distinguish, via (2.5), three mutually exclusive and exhaustive possibilities:

(i) $r_{k+1} \geqq r_{k+1}^{+}(\epsilon), r_{k+2} \leqq r_{\bar{k}+2}(\epsilon)$,

(ii) $r_{k+1} \leqq r_{\overline{k+1}}(\boldsymbol{\epsilon})$,

(iii) $r_{k+2} \geqq r_{k+2}^{+}(\epsilon)$.

If (i) holds, (2.2) and (i) imply 


$$
\overline{r_{k+2}} \geqq r_{k+2} \geqq \frac{k+2}{k}\left[1-\frac{\epsilon}{r_{k+1}^{+}}\right] .
$$

Substituting (2.7) for $j=k$ into (2.8) and recalling the definitions of $r_{k^{+}+1}^{+}, r_{\bar{k}+2}$, we find that

$$
\begin{aligned}
& \frac{k+2}{2 k}\left[1-\left(1-4 \epsilon k(k+2)^{-1}\right)^{1 / 2}\right] \\
& \quad \geqq r_{k+2} \geqq \frac{k+2}{k}\left[1-\frac{k+2}{2 k}\left(1-\left(1-4 \epsilon k(k+2)^{-1}\right)^{1 / 2}\right)\right]
\end{aligned}
$$

which is valid only if $\epsilon \geqq(k+2)^{2} / 4(k+1)^{2}=(n+3)^{2} / 4(n+1)^{2}=\epsilon_{n}^{\prime}$. We now show that a real solution does exist for $\boldsymbol{\epsilon}=\boldsymbol{\epsilon}_{n}^{\prime}$. When $\boldsymbol{\epsilon}=\boldsymbol{\epsilon}_{n}^{\prime}$, (2.9) yields $r_{k+2}=(k+2) / 2(k+1)$. Substituting this value of $r_{k+2}$ and $\boldsymbol{\epsilon}=\boldsymbol{\epsilon}_{n}^{\prime}$ back in $(2.2)$, we find $r_{k+1} \leqq(k+2) / 2(k+1)$ which combined with (i) $r_{k+1} \geqq r_{k+1}^{+}\left(\epsilon_{n}^{\prime}\right)=(k+2) / 2(k+1)$ implies

$$
r_{k+1}=(k+2) / 2(k+1)=r_{k+2} .
$$

But then, as remarked earlier, $b_{j}=(k+2) / 2(k+1)=(n+3) / 2(n+1)$ $=b_{n}^{\prime}$ (say), $j=1,2 \cdots n$ implying $r_{j}=(n+3) / 2(n+1), j=1,2, \cdots n$ and these values of $r_{j}$ are quite compatible with (2.2), (2.2)' and (2.3). Consequently, ${ }^{2}\left(\epsilon_{n}^{\prime}, \bar{b}_{n}^{\prime}\right)$ is a real solution and among all real solutions conforming to (i), it is the one having minimal $\epsilon$.

Since we are concerned only with the possibility of solutions with smaller $\epsilon$, we assume in what follows that $\epsilon \leqq \epsilon_{n}{ }^{\prime}$. Suppose next that (ii) $r_{k+1} \leqq r_{\bar{k}+1}$ prevails. Part (a) of $B$ yields $r_{k} \leqq r_{\bar{k}+1}$. But then necessarily $r_{k} \leqq r_{k}^{-}$since the only alternative permitted by (2.5), namely $r_{k} \geqq r_{k}^{+}$entails (by proposition A) $r_{k} \geqq r_{k}^{+}>r_{\bar{k}+1}$, a contradiction. Repeating this chain of argument, we eventually deduce $r_{2} \leqq r_{2}^{-}$. Applying (a) of $B$ once more, we find $r_{1} \leqq r_{2}^{-}=[1 / n-1]\left[1-\left(1-2 \epsilon_{n}^{\prime}(n-1) n^{-1}\right)^{1 / 2}\right]$ which contradicts $(2.2)^{\prime}$ for $n \geqq 3$.

Finally, the contingency (iii) $r_{k+2} \geqq r_{k+2}^{+}$implies by (b) of $B$ that $r_{k+3}>r_{\bar{k}+3}$. But then (2.5) insures that $r_{k+3} \geqq r_{k+3}^{+}$. Continuing in this fashion, we find that $r_{n} \geqq r_{n}^{+}>n \epsilon$ (for $\left.\epsilon \leqq \epsilon_{n}^{\prime}\right)$ contradicting $(2.2)^{\prime}$ for $n>1$.

When $n=1$, it is trivial to verify that $\epsilon=\epsilon_{1}^{\prime}=1, b=b_{1}^{\prime}=1$ is the unique minimal real solution.

Thus, when $n=2 k+1, P_{n+2}(x)=\left(x^{2}-x+\epsilon\right) \prod_{i=1}^{n}\left(x+b_{i}\right) \in \mathcal{P}^{+}$only if $\epsilon \geqq \epsilon_{n}^{\prime}$. Further, when $\epsilon=\epsilon_{n}^{\prime}$ there is only one possible choice of $b$, namely, $b_{1}=b_{2}=\cdots=b_{n}=\left(\epsilon_{n}^{\prime}\right)^{1 / 2}$. These values cause the central terms of $P_{n+2}(x)$ of degree $k+1$ and $k+2$ to vanish.

${ }^{2}$ It is to be understood wherever a real solution is concerned that $(\epsilon, z)$ abbreviates the $(n+1)$-tuple $(\epsilon, z, z \cdots z)$. 
We turn now to the case $n=2 k, k$ a positive integer, and distinguish four mutually exclusive and exhaustive possibilities:

(i) $r_{k} \geqq r_{k}^{+}(\boldsymbol{\epsilon}), r_{k+1} \leqq r_{\bar{k}+1}(\boldsymbol{\epsilon})$;

(ii) $r_{k+1} \geqq r_{k+1}^{+}(\epsilon), r_{k+2} \leqq r_{\bar{k}+2}(\epsilon)$;

(iii) $r_{k} \leqq r_{\bar{k}}(\epsilon)$,

(iv) $r_{k+2} \geqq r_{k}^{+}+2(\epsilon)$.

Under case (i), employing (2.2) and then (2.7) for $j=k$, we have

$$
\begin{aligned}
-r_{k+1}(\epsilon) \geqq r_{k+1} & \geqq \frac{k+1}{k}\left[1-\frac{\epsilon k}{(k+1) r_{k}^{+}}\right] \\
& =\frac{k+1}{k}\left[1-\frac{k+2}{k-1} r_{k}^{-}\right]
\end{aligned}
$$

which is valid only when $\epsilon \geqq(k+2) / 4 k=(n+4) / 4 n=\epsilon_{n}^{\prime \prime}$ (say). We show next that a solution exists for $\epsilon=\epsilon_{n}^{\prime \prime}$.

For this determination of $\epsilon,(2.10)$ implies that $r_{k+1}=1 / 2$. Substituting these values back in (2.2) we find $r_{k} \leqq 1 / 2$, which, combined with (i) $r_{k} \geqq r_{k}^{+}\left(\epsilon_{n}^{\prime \prime}\right)=1 / 2$, yields the result $r_{k}=1 / 2=r_{k+1}$. But then $b_{j} \equiv 1 / 2$ which, in turn, forces $r_{j} \equiv 1 / 2$ and it is readily checked that these latter values satisfy $(2.2)$ and $(2.2)^{\prime}$. Thus, ${ }^{2}\left(\epsilon_{n}^{\prime \prime}, 1 / 2\right)$ is a real solution and no other real solution compatible with (i) permits a smaller choice of $\epsilon$.

In case (ii) $r_{k+1} \geqq r_{k+1}^{+}(\epsilon), r_{k+2} \leqq r_{\bar{k}+2}(\epsilon)$, analogous reasoning again yields $\epsilon \geqq \epsilon_{n}^{\prime \prime}$ but now $r_{k+1}=r_{k+2}=(k+2) / 2 k=(n+4) / 2 n=2 \epsilon_{n}^{\prime \prime}$ and it is easily verified that $\left(\epsilon_{n}^{\prime \prime}, 2 \epsilon_{n}^{\prime \prime}\right)$ also constitutes a real solution. ${ }^{2}$

The remaining cases (iii) $r_{k} \leqq r_{k}^{-}(\boldsymbol{\epsilon})$ and (iv) $r_{k+2} \geqq r_{k}^{+}+2(\boldsymbol{\epsilon})$ may be disposed of, as in the case $n$ odd, by a systematic use of propositions $\mathrm{A}$ and $\mathrm{B}$.

Thus, when $n=2 k, P_{n+2}(x)=\left(x^{2}-x+\epsilon\right) \prod_{i=1}^{n}\left(x+b_{i}\right) \in \mathcal{P}^{+}$only if $\epsilon \geqq \epsilon_{n}^{\prime \prime}$ and when $\epsilon=\epsilon_{n}^{\prime \prime}$, there are two real solutions obtained by choosing $b_{j} \equiv 2 \epsilon_{n}^{\prime \prime}$ or $b_{j} \equiv 1 / 2$. In the former case the coefficients of the terms of degree $k$ and $k+1$ in $P_{n+2}(x)$ are zero while in the latter case the terms $x^{k}$ and $x^{k-1}$ vanish. Note that $1 / 2<2 \epsilon_{n}^{\prime \prime} \leqq 1$ and that $2 \epsilon_{n}^{\prime \prime} \searrow 1 / 2$. Similarly, $1 / 2<\bar{b}_{n}^{\prime}=\left(\epsilon_{n}^{\prime}\right)^{1 / 2} \leqq 1$ and $\bar{b}_{n}^{\prime} \searrow 1 / 2$.

Define

$$
\begin{aligned}
& \epsilon_{n}=\epsilon_{n}^{\prime}, n \text { odd } \bar{b}_{n}=\bar{b}_{n}=\left(\epsilon_{n}^{\prime}\right)^{1 / 2}, n \text { odd } \\
&=\epsilon_{n}^{\prime \prime}, n \text { even } \bar{b}_{n}=2 \epsilon_{n}^{\prime \prime}, n \text { even } \\
& \overline{\bar{b}}_{n}=1 / 2, n \text { even. }
\end{aligned}
$$

Since $\epsilon_{n}^{\prime}>\epsilon_{n+1}^{\prime \prime}$ and $\epsilon_{n}^{\prime \prime}>\epsilon_{n+1}^{\prime}$, the sequence $\left\{\epsilon_{n}\right\}$ is monotone decreasing (to $1 / 4$ ). Hence, the step function (take $\epsilon_{0}=+\infty$ )

$$
N_{1}(\epsilon)=j, \quad \epsilon_{j} \leqq \epsilon<\epsilon_{j-1}, \quad j=1,2 \cdots
$$


gives the answer to the question initially posed.

Clearly, if $\epsilon^{\prime}>\epsilon$ and $P_{n+2}(x ; \epsilon)=\left(x^{2}-x+\epsilon\right) \coprod_{i=1}^{n}\left(x+b_{i}\right) \in \mathcal{P}^{+}$, then $P_{n+2}\left(x ; \epsilon^{\prime}\right)=P_{n+2}(x ; \epsilon)+\left(\epsilon^{\prime}-\epsilon\right) \prod_{i=1}^{n}\left(x+b_{i}\right) \in \mathcal{P}^{+}$. Consequently, $\left(x^{2}-x+\epsilon\right)\left(x+\bar{b}_{n}\right)^{n} \in \mathcal{P}^{+}$for all $\epsilon \geqq \epsilon_{n}$ and likewise $\left(x^{2}-x+\epsilon\right)\left(x+\bar{b}_{n}\right)^{n}$ $\in \mathcal{P}^{+}, \epsilon \geqq \epsilon_{n}$. Thus, the answer to the second question raised is affirmative. The real numbers $b_{1}, b_{2} \cdots b_{n}$ may always be selected equal without increasing $n$ and indeed when $\epsilon=\epsilon_{n}$, they must be chosen equal if $n$ is to be kept minimal. Expressed otherwise, $N_{1}(\epsilon)=N_{2}(\epsilon)$.

3. Remarks. In this section, we give a more precise sufficient condition than that contained in Theorem 1 of [2] which states that a necessary and sufficient condition that there exist $P_{m}(x)=\sum_{j=0}^{m} a_{j} x^{j}$ $=\left(x^{2}-x+\epsilon\right) P_{m-2}(x) \in \mathcal{P}^{+}$is that $m \geqq M(\epsilon)$ where $M(\epsilon)$ is equal to the smallest integer $j$ for which $j$ arc $\cos \left(2(\epsilon)^{1 / 2}\right)^{-1} \geqq \pi$. Here, $m-2$ and $M(\epsilon)-2$ correspond respectively to the $n$ and $N_{1}(\epsilon)$ of $\S 1$. We employ the notation and results of [2].

Analogous to (2.1) (of $\S 2$ or of [2]), we have (take $P_{m-2}(x)$ $\left.=\sum_{j=0}^{m-2} c_{j} x^{j}\right)$

$$
c_{j-2}-c_{j-1}+\epsilon c_{j}=a_{j} \geqq 0, \quad j=0,1 \cdots m
$$

as a necessary and sufficient condition that $P_{m}(x) \in \mathcal{P}^{+}$. Here, $c_{-2}=c_{-1}$ $=c_{m-1}=c_{m}=1-c_{m-2}=0$.

Multiply (3.1) by $\bar{g}_{j-1}=\bar{g}_{j-1}(\epsilon)$ (see [2] for definition) and sum from $j=2$ to $j=i$ obtaining (via (1.6) of [2] and $\bar{g}_{1}=\bar{g}_{2}=1$ )

$$
c_{0}-\left(\bar{g}_{i-1}-\epsilon \bar{g}_{i-2}\right)+\epsilon \bar{g}_{i-1} c_{i}=\sum_{j=2}^{i} a_{j} \bar{g}_{j+1} .
$$

Hence, from (1.3) of [2],

$$
c_{i} \geqq \frac{\bar{g}_{i}(\epsilon) c_{i-1}-c_{0}}{\epsilon \bar{g}_{i-1}(\epsilon)} \quad \text { for } i<M(\epsilon)-1 .
$$

In analogous fashion, multiply (3.1) by $\epsilon^{-(m-2-j)} \bar{g}_{m-1-j}$ and sum from $j=m-2$ to $j=m-i$ to obtain

$$
\begin{gathered}
\epsilon-\epsilon^{-(i-2)}\left[\bar{g}_{i-1}-\epsilon \bar{g}_{i-2}\right] c_{m-i-1}+\epsilon^{-(i-2)} \bar{g}_{i-1} c_{m-i-2} \\
=\sum_{r=2}^{i} \epsilon^{-(r-2)} \bar{g}_{r-1} a_{m-r},
\end{gathered}
$$

implying

$$
c_{j} \geqq \frac{\bar{g}_{m-j-2} c_{j+1}-\epsilon^{m-j-3}}{\bar{g}_{m-j-3}} \text { for } m-j<M(\epsilon)-1 .
$$


Now, the trigonometric representation (see (1.3) of [2]) of $g_{j}(\delta)$ readily yields the identity $g_{j}^{2}(\delta)-(1+\delta)^{j-1}=g_{j-1}(\delta) g_{j+1}(\delta)$ or equivalently

$$
\bar{g}_{j}^{2}(\epsilon)-\epsilon^{j-1}=\bar{g}_{j-1}(\epsilon) \bar{g}_{j+1}(\epsilon), \quad j \geqq 2 .
$$

Then, utilizing (3.6), an induction on (3.3) gives

$$
c_{j} \geqq \epsilon^{-j} \bar{g}_{j+1}(\epsilon) c_{0},
$$

$$
1 \leqq j<M(\epsilon)-1 .
$$

Similarly, induction on (3.5) leads to

$$
c_{m-j} \geqq \bar{g}_{j-1}(\epsilon), \quad m-j<M(\epsilon)-1 .
$$

Thus, (3.7) and (3.8) furnish simple necessary conditions for the coefficients $c_{j}$ in terms of the known polynomials $\bar{g}_{j}(\boldsymbol{\epsilon})$.

Finally, we note that choosing $c_{j}$ equal to the lower bound of (3.8) gives

$$
\begin{aligned}
P_{m}(x)=\left(x^{2}-x+\epsilon\right) \sum_{i=0}^{m-2} \bar{g}_{m-i-1}(\epsilon) x^{i} & =x^{m}+\left(\epsilon \bar{g}_{m-2}-\bar{g}_{m-1}\right) x+\epsilon \bar{g}_{m-1}(\epsilon) \\
& =x^{m}-\bar{g}_{m}(\epsilon) x+\epsilon \bar{g}_{m-1}(\epsilon) .
\end{aligned}
$$

But for $m=M(\epsilon)$ (as well as for a range of larger values), $g_{m}(\epsilon) \leqq 0$ whence $P_{m}(x) \in \mathcal{P}^{+}, m=M(\epsilon)$.

A trinomial of this form was constructed in the sufficiency proof of Theorem 1 of [2] but the preceding gives the nonvanishing coefficients as explicit functions of $\epsilon$ and at the same time specifies the coefficients of $\left(x^{2}-x+\epsilon\right)^{-1} P_{m}(x)$.

\section{REFERENCES}

1. P. Slepian, Research problem 1, Bull. Amer. Math. Soc. vol. 64 (1958) p. 59.

2. H. Teicher, Reducibility of positive type polynomials, Proc. Amer. Math. Soc. vol. 6 (1955) pp. 195-201.

3. J. V. Uspensky, Theory of equations, McGraw-Hill, New York, 1948.

Purdue University 